\title{
Empleo de modelos de membrana lipídica para el estudio de la función de la proteína SP-C del surfactante pulmonar
}

\author{
Azucena González-Horta ${ }^{\mathrm{a} *}$, María Teresa Dávalos Romo ${ }^{\mathrm{b}}$ y Jesús Pérez-Gil \\ ${ }^{a}$ Universidad Autónoma de Nuevo León, UANL, FCB, Av. Universidad s/n Ciudad Universitaria San Nicolás de los Garza N.L. \\ ${ }^{b}$ Universidad Autónoma de Zacatecas, Campus Jalpa, Jardín Juárez 147 Centro Histórico, Zacatecas \\ ${ }^{c}$ Universidad Complutense de Madrid, Depto. Bioquímica y Biología Molecular, Facultad de Ciencias Biológicas \\ *E-mail: azucena.gonzalezhr@uanl.edu.mx
}

Recibido 5 de noviembre de 2012, Aceptado 3 de diciembre de 2012

\section{Resumen}

El objetivo que se planteó para este trabajo fue entender a nivel molecular la capacidad que tiene la proteína SP-C surfactante pulmonar para modular las propiedades biofísicas del surfactante. Se sintetizaron dos péptidos correspondientes a la secuencia del segmento $\mathrm{N}$-terminal de la proteína, uno con las cisteínas libres y otro con las cisteínas palmitoiladas, para caracterizar el modo y la extensión de la interacción de los péptidos con monocapas fosfolipídicas modelo con especial énfasis, en el análisis del efecto que la palmitoilación de este segmento tiene en la estructura y dinámica interfacial.

Palabras clave: surfactante pulmonar, propiedades tensioactivas, SP-C

\section{Introducción}

Se han identificado 3 modelos de membrana biológica para evaluar la interacción entre ésta y compuestos bioactivos. El primero de ellos, las monocapas de tipo Langmuir, que son un excelente sistema modelo para estudiar la biofísica de membranas en la interfase aire-líquido [1-4]. El segundo, los liposomas, que son sistemas de autoensamblaje compuestos normalmente de moléculas fosfolipídicas que permiten caracterizar la interacción con péptidos y/o proteínas [5-7] y finalmente, las bicapas lipídicas en soporte sólido (tipo Langmuir-Blodgett) que son un sistema que permite reproducir la termodinámica de las membranas celulares y la coexistencia de fases $[8$, 9].

En el presente trabajo se emplearon las monocapas de tipo Langmuir y las de Langmuir-Blodgett para evaluar las propiedades biofísicas de la proteína SP$\mathrm{C}$ en el surfactante pulmonar, una compleja mezcla lipoproteica que forma una fina capa tensioactiva en la interfase aire-líquido previniendo el colapso alveolar [10-12]. El principal componente del surfactante es la dipalmitoilfosfatidilcolina (DPPC) que representa cerca de un $40 \%$ del peso total [12] siendo el responsable de las propiedades tensioactivas de este material [13]. El surfactante cuenta además con cuatro proteínas específicas: SPA y SP-D proteína hidrofilicas implicadas en el sistema de defensa innata del epitelio pulmonar [14, 15] y SP-B y SP-C proteínas hidrofóbicas que interaccionan fuertemente con los lípidos $\mathrm{y}$ promueven la formación y el adecuado comportamiento dinámico de la película tensioactiva en la interfase aire-líquido $[11,15]$.

La proteína SP-C del surfactante, es una proteína biofísicamente activa de 35 aminoácidos que es sintetizada exclusivamente en los neumocitos tipo II Estructuralmente contiene una $\alpha$-hélice transmembrana y un segmento $\mathrm{N}$-terminal catiónico con dos cisteínas palmitoiladas. La mayor parte de las funciones atribuidas a la SP-C en el surfactante están relacionadas con el establecimiento de interacciones bicapa-monocapa que facilitan la transferencia de material tensioactivo durante la dinámica respiratoria, ya sea promoviendo la adsorción interfacial de fosfolípidos y estabilizando la monocapa durante los ciclos dinámicos de compresión-expansión o bien participando en la formación del reservorio superficial de material tensioactivo a partir del cual se restituyen las moléculas de surfactante de la película tensioactiva durante los sucesivos ciclos respiratorios [13-1 Con el fin de fue entender a nivel molecular la capacidad que tiene la proteína SP-C del surfactante pulmonar para modular las propiedades biofísicas del surfactante. Se sintetizaron dos péptidos correspondientes a la secuencia del segmento $\mathrm{N}$ terminal de la proteína, uno con las cisteínas libres (LRIPCCPVNLKRL) y otro con las cisteínas palmitoiladas $\quad\left(\right.$ LRIPC $\left._{\mathrm{Palm}} \mathrm{C}_{\mathrm{Palm}} \mathrm{PVNLKRL}\right)$, para caracterizar el modo y la extensión de la interacción de los péptidos con monocapas fosfolipídicas modelo con especial énfasis, en el análisis del efecto que la palmitoilación de este segmento tiene en la estructura y dinámica interfacial.

\section{Parte Experimental}




\subsection{Compresión dinámica de monocapas}

Para estos experimentos se utilizó una balanza de superficies NIMA de $250 \mathrm{~mL}$ de capacidad provista con una cinta móvil que permite modificar el área que encierra. Se prepararon monocapas de cada uno de los péptidos y se sometieron a compresión dinámica registrando los cambios en la presión superficial. Las muestras peptídicas ó lipopeptídicas se prepararon mezclando las cantidades adecuadas en $\mathrm{Clf} / \mathrm{MetOH}(3: 1 \mathrm{v} / \mathrm{v})$. Para formar las monocapas, se depositaron sobre la subfase (tampón Tris $5 \mathrm{mM}$, $\mathrm{NaCl} 150 \mathrm{mM}$ pH 7) entre 20 y $30 \mu \mathrm{L}$ de muestra dejando equilibrar la monocapa durante $10 \mathrm{~min}$ para permitir la evaporación del disolvente orgánico y la reestructuración de los lípidos en la interfase. Posteriormente la monocapa se comprimió desde un área máxima de $212 \mathrm{~cm}^{2}$ hasta un área de $40 \mathrm{~cm}^{2}$ a una velocidad de $65 \mathrm{~cm}^{2} / \mathrm{min}$ registrando los cambios en la presión superficial.

\subsection{Monocapas y microscopía de epifluorescencia}

Se prepararon monocapas de DPPC/DPPG (7:3 $\mathrm{p} / \mathrm{p}$ ) en ausencia o presencia de 2,5 y $10 \%$ en peso de cada uno de los péptidos. Para la realización de estos experimentos se incluyó en las muestras lipídicas o lipopeptídicas un $1 \%$ de la sonda lipídica fluorescente NBD-PC. Se prepararon muestras de DPPC/DPPG $(7: 3 \mathrm{p} / \mathrm{p})$ a una concentración de 1 $\mathrm{mg} / \mathrm{mL}$ en $\mathrm{Clf} / \mathrm{MetOH}(3: 1 \mathrm{v} / \mathrm{v})$ en ausencia o presencia de un $10 \%$ en peso de péptido y se formó la monocapa como se describió anteriormente. Una vez evaporado el disolvente se comprimió la monocapa hasta una presión inicial entre 3 y 5 $\mathrm{mN} / \mathrm{m}$, dejándola estabilizar por $15 \mathrm{~min}$. Posteriormente se comprimió la superficie de manera continua a una velocidad de $25 \mathrm{~cm}^{2} /$ min mientras se elevaba simultáneamente un portaobjetos previamente sumergido en la subfase a una velocidad de $5 \mathrm{~mm} / \mathrm{min}$, permitiendo así la transferencia de la monocapa al soporte de vidrio, éstos se guardaron en placas de Petri cubiertas con papel aluminio hasta ser analizadas con el microscopio de fluorescencia. Para la cuantificación de las regiones expandidas y condensadas presentes en cada muestra, se tomaron y digitalizaron 5 imágenes para cada presión superficial muestreadas a lo largo del soporte de vidrio cada $2 \mathrm{~mm}$. Las imágenes se analizaron utilizando los programas SigmaScan Pro y AdobePhotoShop.

\section{Resultados}

\subsection{Empleo de monocapas de tipo Langmuir}

El efecto de los péptidos en el comportamiento interfacial de las películas lipopeptídicas se analizó mediante isotermas de compresión las cuales, en el caso de los péptidos puros, proporcionan información sobre su estructura y disposición interfacial. Dos datos importantes pueden obtenerse de estas gráficas. En primer lugar, el área por residuo a la cual la compresión comienza a inducir un incremento en la presión superficial, da idea de lo que ocupa cada molécula de péptido en la disposición más extendida. Por otra parte, la máxima presión a la que puede comprimirse la película peptídica, indica el grado de estabilidad del péptido en la conformación más comprimida. La figura 1 muestra el comportamiento interfacial de monocapas exclusivamente peptídicas sujetas a compresión. Ambos péptidos, con cisteínas libres o palmitoiladas, tienen la capacidad de formar monocapas estables sobre la superficie acuosa como consecuencia de su carácter anfipático. Puede observarse como en ausencia de la acilación, la secuencia N-terminal de SP-C presenta una disposición interfacial mucho más extendida (21-22 $\mathrm{A}^{2} /$ residuo vs 6-7 $\mathrm{A}^{2} /$ residuo). La monocapa de péptido con cisteínas libres colapsa aproximadamente a $24 \mathrm{mN} / \mathrm{m}$, mientras que el péptido palmitoilado puede mantener monocapas a presiones superficiales mayores, en torno a 46 $\mathrm{mN} / \mathrm{m}$. La isoterma del péptido palmitoilado presenta un "plateau" a alrededor de $30 \mathrm{mN} / \mathrm{m}$ antes de alcanzar las mayores presiones superficiales lo que indica la expulsión de la interfase de los segmentos peptídicos. La transición interfacial del péptido palmitoilado conduciría a una película en la que, tras la expulsión de la mayor parte de péptido, permanecerían orientadas hacia el aire las cadenas de palmítico, permitiendo todavía alcanzar presiones superficiales de casi $50 \mathrm{mN} / \mathrm{m}$. Este resultado confirma el papel que tiene la palmitoilación para estabilizar la asociación de la secuencia $\mathrm{N}$-terminal de la SP-C en la interfase, en estados de elevada compresión.
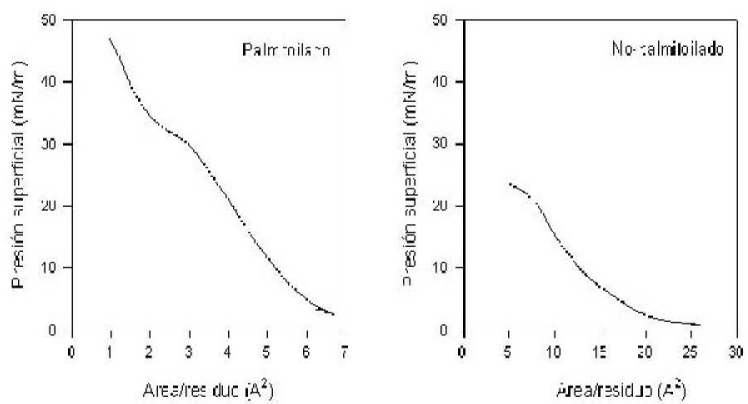

Figura 1. Isotermas de compresión de monocapas de cada uno de los péptidos formadas en ausencia de lípido. 


\subsection{Empleo de monocapas de tipo Langmuir- Blodgett}

Para analizar los efectos que la interacción lípidopéptido tiene sobre el empaquetamiento $\mathrm{y}$ organización de las moléculas lipídicas en la interfase, se transfirieron monocapas de DPPC/DPPG 7:3 (p/p) en ausencia o presencia de cada uno de los péptidos a un soporte sólido y se analizaron por microscopía de epifluorescencia. En la figura 2 puede observarse como ambos péptidos producen un aumento en el tamaño de los dominios y reducen su número debido a que el establecimiento de interacciones electrostáticas permite una mayor condensación favoreciendo la nucleación de regiones condensadas.

El péptido palmitoilado muestra un efecto bifásico, por debajo de $\sim 18 \mathrm{mN} / \mathrm{m}$ el péptido no perturba el empaquetamiento lipídico de la monocapa como se deduce del tamaño y número de los dominios observados en las imágenes de epifluorescencia. Sin embargo a presiones mayores, el péptido produce una drástica disminución del área total ocupada por fase condensada lo que ocurre posiblemente como consecuencia de la inserción de los palmíticos en las regiones más condensadas de la monocapa una vez que la región peptídica ha sido expulsada de la interfase. Estos resultados sugieren que durante la compresión, los segmentos peptídicos son expulsados de la interfase mientras que el péptido palmitoilado mantiene la interacción de los palmíticos en las películas interfaciales altamente comprimidas.
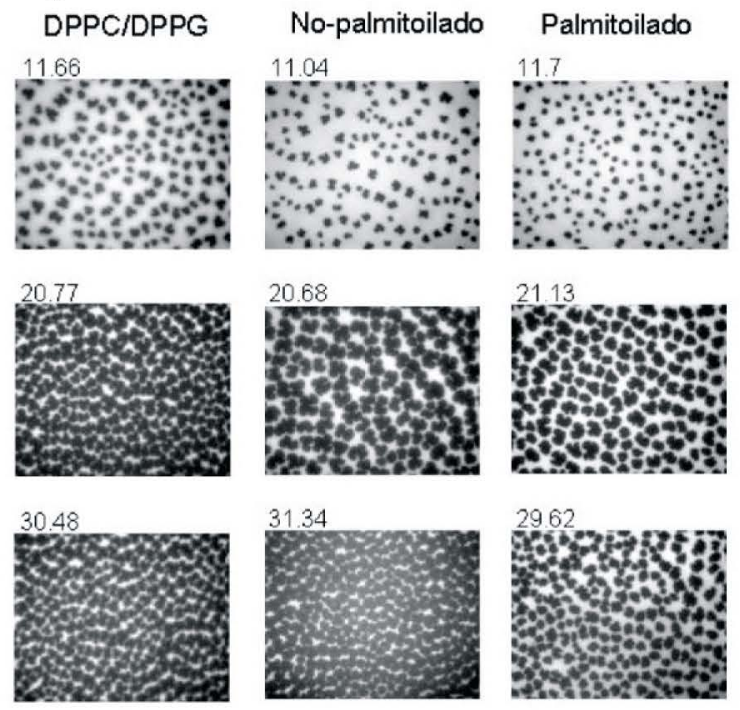

Figura 2. Imágenes de epifluorescencia de monocapas de DPPC/DPPG 7:3 (p/p) comprimidas hasta las presiones indicadas $(\mathrm{mN} / \mathrm{m})$ en ausencia o presencia de $10 \%$ en peso de las secuencias peptídicas empleadas. Cada imagen cubre una distancia de $350 \mu \mathrm{m}$.

\section{Discusión}

Se ha demostrado en numerosas ocasiones que la proteína SP-C del surfactante pulmonar es capaz por sí sola de promover la inserción de moléculas fosfolipídicas desde los complejos lipoproteicos en que el surfactante es secretado a la interfase aire líquido [16-18]. El segmento transmembranal de la SP-C es fundamental para la función de la proteína, sin embargo algunas características funcionales esenciales de la SP-C pueden residir en las interacciones lípido-proteína en las que participa el segmento $\mathrm{N}$-terminal, especialmente si se considera que este segmento puede ser la única parte de la estructura que la SP-C proyecta hacia el exterior de las membranas y monocapas de surfactante [19]. El segmento N-terminal de la SP-C inicia posiblemente su contacto con la interfase aire-líquido durante el proceso de adsorción de los complejos lipoproteicos del surfactante a la interfase. Este proceso de adsorción debe estar necesariamente relacionado con la perturbación que el segmento $\mathrm{N}$-terminal es capaz de producir en el empaquetamiento lipídico de bicapas y monocapas [20,21]. La mayor parte de los modelos planteados sobre la estructura y disposición de la SP-C en las bicapas y monocapas de surfactante suponen que el segmento $\mathrm{N}$-terminal de la proteína se asocia con las membranas a través de la palmitoilación de sus cisteínas [22]. Sin embargo, al realizar las cinéticas de adsorción interfacial de los péptidos (datos no mostrados) pudo observarse que la inclusión del péptido palmitoilado en membranas fosfolipídicas no resulta muy eficiente para promover la transferencia de especies fosfolipídicas tensioactivas desde las bicapas a la interfase. La diferencia en actividad debe necesariamente estar relacionada con la diferencia que introduce la palmitoilación en la extensión y modo de interacción del segmento N-terminal con las superficies lipídicas. La adsorción interfacial de fosfolípidos desde las bicapas probablemente requiere la perturbación simultánea del empaquetamiento lipídico en bicapas y monocapa. Esta perturbación podría ser más accesible al péptido desacilado, con una conformación aparentemente más extendida, que al péptido palmitoilado. La eficiente actividad interfacial de la SP-C nativa, a pesar de poseer su segmento N-terminal estequiométricamente palmitoilado, podría depender de la simultánea presencia del segmento helicoidal, que manteniéndose integrado en la membrana permite el acercamiento y perturbación por parte del segmento N-terminal en una monocapa adyacente. Sin embargo, una vez en la película interfacial, el péptido palmitoilado resulta asociarse con la interfase de una manera más estable que el péptido 
no palmitoilado, como había sido propuesto mediante estudios de IRRAS [23], manteniéndose a presiones mucho mayores que las necesarias para expulsar el péptido desacilado.

\section{Conclusión}

Los experimentos aquí presentados demuestran que el empleo de modelos de membrana es útil para evaluar las propiedades biofísicas de una proteína de interés. En este caso en particular, los resultados sugieren que durante los ciclos de compresión del material tensioactivo, primero son expulsados los segmentos peptídicos de la proteína SP-C para posteriormente permitir su integración a través de la intercalación de los palmíticos en las regiones hidrofóbicas altamente empaquetadas de los fosfolípidos comprimidos de las películas interfaciales. Esta asociación podría entonces mantenerse hasta las mayores presiones superficiales y ser consistente con un papel de la palmitoilación en la capacidad de la SP-C para sostener la asociación de los complejos lipoproteicos del surfactante con las fases altamente comprimidas que se forman al final de la espiración, facilitando su posterior reextensión tras cada ciclo respiratorio.

\section{Referencias}

1. Kaganer V.M., Möhwald H., Dutta P. Rev Mod Phys. 1999, 71, 779-819.

2. Brezesinski G., Möhwald H., Adv Colloid Inteface Sci. 2003 , 100-102, 563-84.

3. Mälkiä A., Liljeroth P., Kontturi K. Anal Sci. 2001, 17, 34548.

4. Gambinossi F., Mecheri B., Nocentini M., Puggelli M., Caminati G. Biophys Chem. 2004, 110, 101-17.

5. Park J.W. Colloids Surf B Bionterfaces 2010, 75, 290-3.

6. Parry MJ., Jutila A., Kinnunen PK., Alakoskela JM. 2007, 17, 97-103.

7. Jorgensen K., Hoyrup P., Pedersen TB., Mouritsen OG. 2001, 6, 255-63.

8. Tamm LK:, McConnell HM: Biophys J. 1985, 47, 105-13.

9. Kiesslig V., Domanska MK., Murray D., Wan C., Tamm LK:, Wiley Encyclopedia of Chemical Biology. USA: John Wiley and Sons, 2008. Supported Lipid Bilayers: Development and Applications in Chemical Biology.

10. Daniels, D.B. and S. Orgeig, News Physiol Sci 2003, 18: 151175 .

11. Goerke, J. Biochim Biophys Acta, 1998. 1408: 79-89.

12. Veldhuizen, J.A. and H.P. Haagsman, Biochim Biophys Acta 2000, 1467, 255-270.

13. Van Golde, L.M.G. and Casals, C. 1997. Philadelphia WJ Crustal RG, Wibel ER, Barnes PJ Lippincott-Raven Publishers.

14. Lawson, R.R. and Reid, K.B. Immunol Rev. 2000, 173, 66-78.

15. Possmayer F., Nag K., Rodriguez K., Qanbar R., Shürch S. Comp Biochem Physiol 2001. 129: 209-220.

16. Perez-Gil J., Nag K., Taneva S., Keough KMW. Biophys $J$ 1992, 63, 197-204.

17. Lukovic D., Plasencia I., Taberner FJ ; Salgado J., Calvete JJ, Perez-Gil J., Mingarro I. Biochim Biophys Acta 2006, 1758 (4), 509-518.
18. Oosterlaken-Dijksterhuis MA, van Eijk M, van Buel BL, van Golde LM, Haagsmann HP. Biochem J 1991, 273, 115-119.

19. Plasencia I, Cruz A, Casals C, Perez-Gil J. Biochem J 2001, 359, 651-659.

20. Plasencia I, Keough K.M.W., Perez-Gil J. Biochim Biophys Acta 2005, 1713, 118-128.

21. Plasencia I., Rivas L., Keough KM., Marsh D., Perez-Gil J. Biochim J 2004, 377, 183-193.

22. Johansson, J. Biochim Biophys Acta 1998, 1408, 161-172.

23. Bi, X., Flash CR., Perez-Gil J., Plasencia I., Andreu D., Oliveira E., Mendelsohn R. Biochemistry 2002, 41, 83858395. 